\title{
The Number of Symbol Comparisons in QuickSort and QuickSelect
}

\author{
Brigitte VALLÉE ${ }^{1}$, Julien Clément ${ }^{1}$, James Allen Fill ${ }^{2}$, and \\ Philippe FLAJOLET ${ }^{3}$ \\ 1 GREYC, CNRS and University of Caen, 14032 Caen Cedex (France) \\ 2 Applied Mathematics and Statistics, The Johns Hopkins University, \\ Baltimore, MD 21218-2682 (USA) \\ 3 Algorithms Project, INRIA-Rocquencourt, 78153 Le Chesnay (France)
}

\begin{abstract}
We revisit the classical QuickSort and QuickSelect algorithms, under a complexity model that fully takes into account the elementary comparisons between symbols composing the records to be processed. Our probabilistic models belong to a broad category of information sources that encompasses memoryless (i.e., independent-symbols) and Markov sources, as well as many unbounded-correlation sources. We establish that, under our conditions, the average-case complexity of QuickSort is $O\left(n \log ^{2} n\right)$ [rather than $O(n \log n)$, classically], whereas that of QuickSelect remains $O(n)$. Explicit expressions for the implied constants are provided by our combinatorial-analytic methods.
\end{abstract}

Introduction. Every student of a basic algorithms course is taught that, on average, the complexity of Quicksort is $O(n \log n)$, that of binary search is $O(\log n)$, and that of radix-exchange sort is $O(n \log n)$; see for instance $[13,16]$. Such statements are based on specific assumptions - that the comparison of data items (for the first two) and the comparison of symbols (for the third one) have unit cost - and they have the obvious merit of offering an easy-to-grasp picture of the complexity landscape. However, as noted by Sedgewick, these simplifying assumptions suffer from limitations: they do not make possible a precise assessment of the relative merits of algorithms and data structures that resort to different methods (e.g., comparison-based versus radix-based sorting) in a way that would satisfy the requirements of either information theory or algorithms engineering. Indeed, computation is not reduced to its simplest terms, namely, the manipulation of totally elementary symbols, such as bits, bytes, characters. Furthermore, such simplified analyses say little about a great many application contexts, in databases or natural language processing, for instance, where information is highly "non-atomic", in the sense that it does not plainly reduce to a single machine word.

First, we observe that, for commonly used data models, the mean costs $S_{n}$ and $K_{n}$ of any algorithm under the symbol-comparison and the key-comparison model, respectively, are connected by the universal relation $S_{n}=K_{n} \cdot O(\log n)$. (This results from the fact that at most $O(\log n)$ symbols suffice, with high probability, to distinguish $n$ keys; cf. the analysis of the height of digital trees, 
also known as "tries", in [3].) The surprise is that there are cases where this upper bound is tight, as in QuickSort; others where both costs are of the same order, as in QuickSelect. In this work, we show that the expected cost of QuickSort is $O\left(n \log ^{2} n\right)$, not $O(n \log n)$, when all elementary operationssymbol comparisons - are taken into account. By contrast, the cost of QuickSelect turns out to be $O(n)$, in both the old and the new world, albeit, of course, with different implied constants. Our results constitute broad extensions of earlier ones by Fill and Janson (Quicksort, [7]) and Fill and Nakama (QuickSelect, [8]).

The sources that we deal with include memoryless ("Bernoulli") sources with possibly non-uniform independent symbols and Markov sources, as well as many non-Markovian sources with unbounded memory from the past. A central idea is the modelling of the source via its fundamental probabilities, namely the probabilities that a word of the source begins with a given prefix. Our approach otherwise relies on methods from analytic combinatorics [10], as well as on information theory and the theory of dynamical systems. It relates to earlier analyses of digital trees ("tries") by Clément, Flajolet, and Vallée $[3,19]$.

Results. We consider a totally ordered alphabet $\Sigma$. What we call a probabilistic source produces infinite words on the alphabet $\Sigma$ (see Definition 1). The set of keys (words, data items) is then $\Sigma^{\infty}$, endowed with the strict lexicographic order denoted ' $\prec$ '. Our main objects of study are two algorithms, applied to $n$ keys assumed to be independently drawn from the same source $\mathcal{S}$, namely, the standard sorting algorithm QuickSort $(n)$ and the algorithm QuickSelect $(m, n)$, which selects the $m$ th smallest element. In the latter case, we shall mostly focus our attention on situations where the rank $m$ is proportional to $n$, being of the form $m=\alpha n$, so that the algorithm determines the $\alpha$ th quantile; it will then be denoted by QuickQuant ${ }_{\alpha}(n)$. We also consider the cases where rank $m$ equals 1 (which we call QuickMin), equals $n$ (QuickMax), or is randomly chosen in $\{1, \ldots, n\}$ (QuickRand).

Our main results involve constants that depend on the source $\mathcal{S}$ (and possibly on the real $\alpha$ ); these are displayed in Figures 1-3 and described in Section 1. They specialize nicely for a binary source $\mathcal{B}$ (under which keys are compared via their binary expansions, with uniform independent bits), in which case they admit pleasant expressions that simplify and extend those of Fill and Nakama [8] and Grabner and Prodinger [11] and lend themselves to precise numerical evaluations (Figure 2). The conditions $\Lambda$-tamed, $\Pi$-tamed, and "periodic" are made explicit in Subsection 2.1. Conditions of applicability to classical source models are discussed in Section 3.

Theorem 1. (i) For a $\Lambda$-tamed source $\mathcal{S}$, the mean number $T_{n}$ of symbol comparisons of QuickSort $(n)$ involves the entropy $h(\mathcal{S})$ of the source:

$$
T_{n}=\frac{1}{h(\mathcal{S})} n \log ^{2} n+a(\mathcal{S}) n \log n+b(\mathcal{S}) n+o(n), \quad \text { for some } a(\mathcal{S}), b(\mathcal{S}) \in \mathbb{R} . \quad(1)
$$

(ii) For a periodic source, a term $n P(\log n)$ is to be added to the estimate (1), where $P(u)$ is a (computable) continuous periodic function. 
Theorem 2. For a $\Pi$-tamed source $\mathcal{S}$, one has the following, with $\delta=\delta(\mathcal{S})>0$.

(i) The mean number of symbol comparisons $Q_{n}^{(\alpha)}$ for QuickQuant ${ }_{\alpha}$ satisfies

$$
Q_{n}^{(\alpha)}=\rho_{\mathcal{S}}(\alpha) n+O\left(n^{1-\delta}\right)
$$

(ii) The mean number of symbol comparisons, $M_{n}^{(-)}$for QuickMin $(n)$ and $M_{n}^{(+)}$ for QuickMax $(n)$, satisfies with $\epsilon= \pm$,

$$
M_{n}^{(\epsilon)}=\rho_{\mathcal{S}}^{(\epsilon)} n+O\left(n^{1-\delta}\right), \quad \text { with } \quad \rho_{\mathcal{S}}^{(+)}=\rho_{\mathcal{S}}(1), \quad \rho_{\mathcal{S}}^{(-)}=\rho_{\mathcal{S}}(0) .
$$

(iii) The mean number $M_{n}$ of symbol comparisons performed by QuickRand $(n)$ satisfies

$$
M_{n}=\gamma_{\mathcal{S}} n+O\left(n^{1-\delta}\right), \quad \text { with } \quad \gamma_{\mathcal{S}}=\int_{0}^{1} \rho(\alpha) d \alpha .
$$

These estimates are to be compared to the classical ones (see, e.g., [13, p.634] and [12], for extensions), relative to the number of comparisons in QuickQuant ${ }_{\alpha}$ :

$$
K_{n}^{(\alpha)}=\kappa(\alpha) n+O(\log n), \quad \kappa(\alpha):=2(1-\alpha \log (\alpha)-(1-\alpha) \log (1-\alpha)) .
$$

General strategy. We operate under a general model of source, parametrized by the unit interval $\mathcal{I}$. Our strategy comprises three main steps. The first two are essentially algebraic, while the last one relies on complex analysis.

Step $(a)$. We first show (Proposition 1) that the mean number $S_{n}$ of symbol comparisons performed by a (general) algorithm $\mathcal{A}(n)$ applied to $n$ words from a source $\mathcal{S}$ can be expressed in terms of two objects: $(i)$ the density $\phi_{n}$ of the algorithm, which uniquely depends on the algorithm and provides a measure of the mean number of key-comparisons performed near a specific point; (ii) the family of fundamental triangles, which solely depends on the source and describes the location of pairs of words which share a common prefix.

Step $(b)$. This step is devoted to computing the density of the algorithm. We first deal with the Poisson model, where the number of keys, instead of being fixed, follows a Poisson law of parameter $Z$. We provide an expression of the Poissonized density relative to each algorithm, from which we deduce the mean number of comparisons under this model. Then, simple algebra yield expressions relative to the model where the number $n$ of keys is fixed.

Step $(c)$. The exact representations of the mean costs is an alternating sum which involves two kinds of quantities, the size $n$ of the set of data to be sorted (which tends to infinity), and the fundamental probabilities (which tend to 0). We approach the corresponding asymptotic analysis by means of complex integral representations of the Nörlund-Rice type. For each algorithm-source pair, a series of Dirichlet type encapsulates both the properties of the source and the characteristics of the algorithm - this is the mixed Dirichlet series, denoted by $\varpi(s)$, whose singularity structure in the complex plane is proved to condition our final asymptotic estimates.

\section{Algebraic analysis}

The developments of this section are essentially formal (algebraic) and exact; that is, no approximation is involved at this stage. 
Entropy constant [Quicksort, Theorem 1]: $h(\mathcal{S}):=\lim _{k \rightarrow \infty}\left[-\frac{1}{k} \sum_{w \in \Sigma^{k}} p_{w} \log p_{w}\right]$.

Quantile constant $\rho_{\mathcal{S}}(\alpha)$ [QuickQuant, Theorem 2, Part $(i)$ ]

$$
\rho_{\mathcal{S}}(\alpha):=\sum_{w \in \Sigma^{\star}} p_{w} L\left(\frac{\left|\alpha-\mu_{w}\right|}{p_{w}}\right) .
$$

Here, $\mu_{w}:=(1 / 2)\left(p_{w}^{(+)}+p_{w}^{(-)}\right)$involves the probabilities $p_{w}^{(+)}$and $p_{w}^{(-)}$defined in Subsection 1.1; the function $L$ is given by $L(y):=2[1+H(y)]$, where $H(y)$ is expressed by $y^{+}:=(1 / 2)+y, y^{-}:=(1 / 2)-y$, and

$$
H(y):=\left\{\begin{array}{cl}
-\left(y^{+} \log y^{+}+y^{-} \log y^{-}\right), & \text {if } 0 \leq y<1 / 2 \\
0, & \text { if } y=1 / 2 \\
y^{-}\left(\log y^{+}-\log \left|y^{-}\right|\right), & \text {if } y>1 / 2
\end{array}\right.
$$

- Min/max constants [QuickMin, QuickMax, Theorem 2, Part (ii)]:

$$
\rho_{\mathcal{S}}^{(\epsilon)}=\sum_{w \in \Sigma^{\star}} p_{w}\left[1-\frac{p_{w}^{(\epsilon)}}{p_{w}} \log \left(1+\frac{p_{w}}{p_{w}^{(\epsilon)}}\right)\right] .
$$

- Random selection constant [QuickRand, Theorem 2, Part (iii)]:

$$
\gamma_{\mathcal{S}}=\sum_{w \in \Sigma^{\star}} p_{w}^{2}\left[2+\frac{1}{p_{w}}+\sum_{\epsilon= \pm}\left[\log \left(1+\frac{p_{w}^{(\epsilon)}}{p_{w}}\right)-\left(\frac{p_{w}^{(\epsilon)}}{p_{w}}\right)^{2} \log \left(1+\frac{p_{w}}{p_{w}^{(\epsilon)}}\right)\right]\right] .
$$

Figure 1. The main constants of Theorems 1 and 2 , relatively to a general source $(\mathcal{S})$.

$$
\begin{aligned}
& h(\mathcal{B})=\log 2 \quad[\text { entropy] } \\
& \rho_{\mathcal{B}}^{(\epsilon)}=4+2 \sum_{\ell \geq 0} \frac{1}{2^{\ell}} \sum_{k=1}^{2^{\ell}-1}\left[1-k \log \left(1+\frac{1}{k}\right)\right] \doteq 5.279378241080958 . \\
& \gamma_{\mathcal{B}}=\frac{14}{3}+2 \sum_{\ell=0}^{\infty} \frac{1}{2^{2 \ell}} \sum_{k=1}^{2^{\ell}-1}\left[k+1+\log (k+1)-k^{2} \log \left(1+\frac{1}{k}\right)\right] \doteq 8.2073088638 .
\end{aligned}
$$

Figure 2. The constants relative to a binary source.
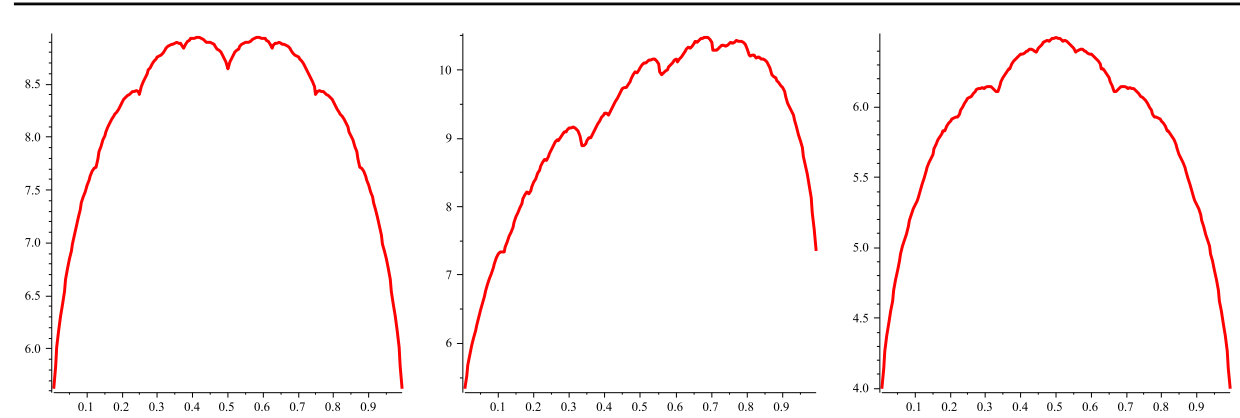

Figure 3. Plot of the function $\rho_{\mathcal{S}}(\alpha)$ for $\alpha \in[0,1]$ and the three sources: $\operatorname{Bern}\left(\frac{1}{2}, \frac{1}{2}\right)$, $\operatorname{Bern}\left(\frac{1}{3}, \frac{2}{3}\right)$, and $\operatorname{Bern}\left(\frac{1}{3}, \frac{1}{3}, \frac{1}{3}\right)$. The curves illustrate the fractal character of the constants involved in QuickSelect. (Compare with $\kappa(\alpha)$ in Eq. (5), which appears as limit of $\rho_{\mathcal{S}}(\alpha)$, for an unbiased $r$-ary source $\mathcal{S}$, when $r \rightarrow \infty$.) 

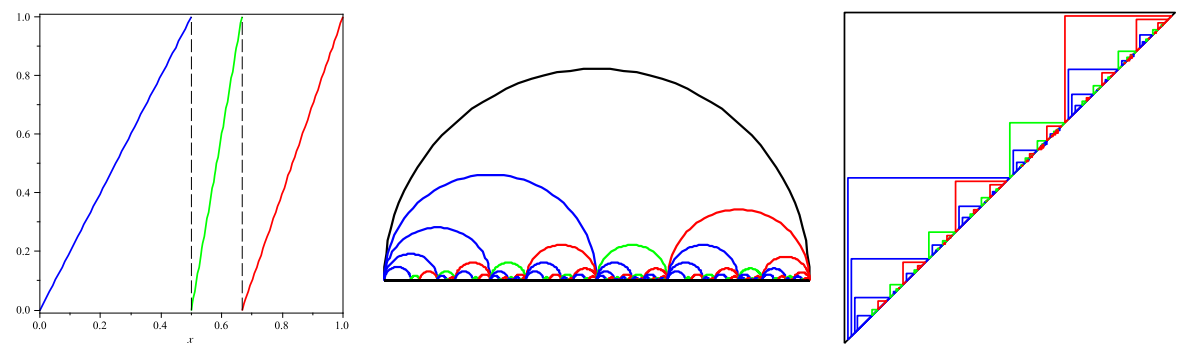

Figure 4. Left: the shift transformation $T$ of a ternary Bernoulli source with $\mathbb{P}(0)=$ $1 / 2, \mathbb{P}(1)=1 / 6, \mathbb{P}(2)=1 / 3$. Middle: fundamental intervals $\mathcal{I}_{w}$ materialized by semicircles. Right: the corresponding fundamental triangles $\mathcal{T}_{w}$.

1.1. A general source model. Throughout this paper, a totally ordered (finite or denumerable) alphabet $\Sigma$ of "symbols" or "letters" is fixed.

Definition 1. A probabilistic source, which produces infinite words of $\Sigma^{\infty}$, is specified by the set $\left\{p_{w}, w \in \Sigma^{\star}\right\}$ of fundamental probabilities $p_{w}$, where $p_{w}$ is the probability that an infinite word begins with the finite prefix $w$. It is furthermore assumed that $\pi_{k}:=\sup \left\{p_{w}: w \in \Sigma^{k}\right\}$ tends to 0 , as $k \rightarrow \infty$.

For any prefix $w \in \Sigma^{\star}$, we denote by $p_{w}^{(-)}, p_{w}^{(+)}, p_{w}$ the probabilities that a word produced by the source begins with a prefix $w^{\prime}$ of the same length as $w$, which satisfies $w^{\prime} \prec w, w^{\prime} \succ w$, or $w^{\prime}=w$, respectively. Since the sum of these three probabilities equals 1 , this defines two real numbers $a_{w}, b_{w} \in[0,1]$ for which

$$
a_{w}=p_{w}^{(-)}, \quad 1-b_{w}=p_{w}^{(+)}, \quad b_{w}-a_{w}=p_{w} .
$$

Given an infinite word $X \in \Sigma^{\infty}$, denote by $w_{k}$ its prefix of length $k$. The sequence $\left(a_{w_{k}}\right)$ is increasing, the sequence $\left(b_{w_{k}}\right)$ is decreasing, and $b_{w_{k}}-a_{w_{k}}$ tends to 0 . Thus a unique real $\pi(X) \in[0,1]$ is defined as common limit of $\left(a_{w_{k}}\right)$ and $\left(b_{w_{k}}\right)$. Conversely, a mapping $M:[0,1] \rightarrow \Sigma^{\infty}$ associates, to a number $u$ of the interval $\mathcal{I}:=[0,1]$, a word $M(u):=\left(m_{1}(u), m_{2}(u), m_{3}(u), \ldots\right) \in \Sigma^{\infty}$. In this way, the lexicographic order on words (' $\prec$ ') is compatible with the natural order on the interval $\mathcal{I}$; namely, $M(t) \prec M(u)$ if and only if $t<u$. Then, the fundamental interval $\mathcal{I}_{w}:=\left[a_{w}, b_{w}\right]$ is the set of reals $u$ for which $M(u)$ begins with the prefix $w$. Its length equals $p_{w}$.

Our analyses involve the two Dirichlet series of fundamental probabilities,

$$
\Lambda(s):=\sum_{w \in \Sigma^{\star}} p_{w}^{-s}, \quad \Pi(s):=\sum_{k \geq 0} \pi_{k}^{-s}
$$

these are central to our treatment. They satisfy, for $s<0$, the relations $\Pi(s) \leq$ $\Lambda(s) \leq \Pi(s+1)$. The series $\Lambda(s)$ always has a singularity at $s=-1$, and $\Pi(s)$ always has a singularity at $s=0$, The regularity properties of the source can be expressed in terms of $\Lambda$ near $s=-1$, and $\Pi$ near 0 , as already shown in [19].

An important subclass is formed by dynamical sources, which are closely related to dynamical systems on the interval; see Figure 4 and [19]. One starts 
with a partition $\left\{\mathcal{I}_{\sigma}\right\}$ indexed by symbols $\sigma \in \Sigma$, a coding map $\tau: \mathcal{I} \rightarrow \Sigma$ which equals $\sigma$ on $\mathcal{I}_{\sigma}$, and a shift map $T: \mathcal{I} \rightarrow \mathcal{I}$ whose restriction to each $\mathcal{I}_{\sigma}$ is increasing, invertible, and of class $\mathcal{C}^{2}$. Then the word $M(u)$ is the word that encodes the trajectory $\left(u, T u, T^{2} u, \ldots\right)$ via the coding map $\tau$, namely, $M(u):=$ $\left(\tau(u), \tau(T u), \tau\left(T^{2} u\right), \ldots\right)$. All memoryless (Bernoulli) sources and all Markov chain sources belong to the general framework of Definition 1: they correspond to a piecewise linear shift, under this angle. For instance, the standard binary system is obtained by $T(x)=\{2 x\}(\{\cdot\}$ is the fractional part). Dynamical sources with a non-linear shift allow for correlations that depend on the entire past (e.g., sources related to continued fractions obtained by $T(x)=\{1 / x\})$.

1.2. The algorithm QuickVal ${ }_{\alpha}$. We consider an algorithm that is dual of QuickSelect: it takes as input a set of words $\mathcal{X}$ and a value $V$, and returns the rank of $V$ inside the set $\mathcal{X} \cup\{V\}$. This algorithm is of independent interest and is easily implemented as a variant of QuickSelect by resorting to the usual partitioning phase, then doing a comparison between the value of the pivot and the input value $V$ (rather than a comparison between their ranks). We call this modified QuickSelect algorithm QuickVal ${ }_{\alpha}$ when it is used to seek the rank of the data item with value $M(\alpha)$. The main idea here is that the behaviors of QuickVal ${ }_{\alpha}(n)$ and QuickQuant ${ }_{\alpha}(n)$ should be asymptotically similar. Indeed, the $\alpha$-quantile of a random set of words of large enough cardinality must be, with high probability, close to the word $M(\alpha)$.

1.3. An expression for the mean number of symbol comparisons. The first object of our analysis is the density of the algorithm $\mathcal{A}(n)$, which measures the number of key-comparisons performed by the algorithm. The second object is the collection of fundamental triangles (see Figure 4).

Definition 2. The density of an algorithm $\mathcal{A}(n)$ which compares $n$ words from the same probabilistic source $\mathcal{S}$ is defined as follows:

$\phi_{n}(u, t) d u d t:=$ "the mean number of key-comparisons performed by

$\mathcal{A}(n)$ between words $M\left(u^{\prime}\right), M\left(t^{\prime}\right)$, with $u^{\prime} \in[u, u+d u]$ and $t^{\prime} \in[t, t+d t] . "$

For each $w \in \Sigma^{\star}$, the fundamental triangle of prefix $w$, denoted by $\mathcal{T}_{w}$, is the triangle built on the fundamental interval $\mathcal{I}_{w}:=\left[a_{w}, b_{w}\right]$ corresponding to $w$; i.e., $\mathcal{T}_{w}:=\left\{(u, t): a_{w} \leq u \leq t \leq b_{w}\right\}$. We set $\mathcal{T} \equiv \mathcal{T}_{\epsilon}:=\{(u, t): 0 \leq u \leq t \leq 1\}$.

Our first result establishes a relation between the mean number $S_{n}$ of symbolcomparisons, the density $\phi_{n}$, and the fundamental triangles $\mathcal{T}_{w}$ :

Proposition 1. Fix a source on alphabet $\Sigma$, with fundamental triangles $\mathcal{T}_{w}$. For any integrable function $g$ on the unit triangle $\mathcal{T}$, define the integral transform

$$
\mathcal{J}[g]:=\sum_{w \in \Sigma^{\star}} \int_{\mathcal{T}_{w}} g(u, t) d u d t
$$

Then the mean number $S_{n}$ of symbol comparisons performed by $\mathcal{A}(n)$ is equal to

$$
S_{n}=\mathcal{J}\left[\phi_{n}\right]=\sum_{w \in \Sigma^{\star}} \int_{\mathcal{T}_{w}} \phi_{n}(u, t) d u d t
$$


where $\phi_{n}$ is the density of algorithm $\mathcal{A}(n)$.

Proof. The coincidence function $\gamma(u, t)$ is the length of the largest common prefix of $M(u)$ and $M(t)$, namely, $\gamma(u, t):=\max \left\{\ell: m_{j}(u)=m_{j}(t), \forall j \leq \ell\right\}$. Then, the number of symbol comparisons needed to compare two words $M(u)$ and $M(t)$, is $\gamma(u, t)+1$ and the mean number $S_{n}$ of symbol comparisons performed by $\mathcal{A}(n)$ satisfies

$$
S_{n}=\int_{\mathcal{T}}[\gamma(u, t)+1] \phi_{n}(u, t) d u d t,
$$

where $\phi_{n}(u, t)$ is the density of the algorithm. Two useful identities are

$$
\sum_{\ell \geq 0}(\ell+1) \mathbf{1}_{[\gamma=\ell]}=\sum_{\ell \geq 0} \mathbf{1}_{[\gamma \geq \ell]} \quad[\gamma \geq \ell]=\bigcup_{w \in \Sigma^{\ell}}\left(\mathcal{I}_{w} \times \mathcal{I}_{w}\right) .
$$

The first one holds for any integer-valued random variable $\gamma\left(\mathbf{1}_{A}\right.$ is the indicator of $\left.A\right)$. The second one follows from the definitions of the coincidence $\gamma$ and of the fundamental intervals $\mathcal{I}_{w}$. Finally, the domain $\mathcal{T} \cap[\gamma \geq \ell]$ is a union of fundamental triangles.

1.4. Computation of Poissonized densities. Under the Poisson model $\mathrm{Poi}_{Z}$ of parameter $Z$, the number $N$ of keys is no longer fixed but rather follows a Poisson law of parameter $Z$ :

$$
\mathbb{P}[N=n]=e^{-Z} \frac{Z^{n}}{n !}
$$

Then, the expected value $\widetilde{S}_{Z}$ in this model, namely,

$$
\widetilde{S}_{Z}:=e^{-Z} \sum_{n \geq 0} \frac{Z^{n}}{n !} S_{n}=e^{-Z} \sum_{n \geq 0} \frac{Z^{n}}{n !} \mathcal{J}\left[\phi_{n}\right]=\mathcal{J}\left[e^{-Z} \sum_{n \geq 0} \frac{Z^{n}}{n !} \phi_{n}\right]=\mathcal{J}\left[\widetilde{\phi}_{Z}\right],
$$

involves the Poissonized density $\widetilde{\phi}_{Z}$, defined as

$$
\widetilde{\phi}_{Z}(u, t):=e^{-Z} \sum_{n \geq 0} \frac{Z^{n}}{n !} \phi_{n}(u, t) .
$$

The following statement shows that the Poissonized densities relative to QuickSort and QuickVal admit simple expressions, which in turn entail nice expressions for the mean value $\widetilde{S}_{Z}$ in this Poisson model, via the equality $\widetilde{S}_{Z}=\mathcal{J}\left[\widetilde{\phi}_{Z}\right]$.

Proposition 2. Set $f_{1}(\theta):=e^{-\theta}-1+\theta$. The Poissonized densities of QuickSort and QuickVal ${ }_{\alpha}$ satisfy

$$
\begin{aligned}
& \widetilde{g}_{Z}(u, t)=2(t-u)^{-2} f_{1}(Z(t-u)), \\
& \widetilde{g}_{Z}^{(\alpha)}(u, t)=2(\max (\alpha, t)-\min (\alpha, u))^{-2} f_{1}(Z(\max (\alpha, t)-\min (\alpha, u)) .
\end{aligned}
$$

The mean number of comparisons of QuickSort and QuickVal ${ }_{\alpha}$ in the Poisson model satisfy

$$
\begin{aligned}
& \widetilde{S}_{Z}=2 \mathcal{J}\left[(t-u)^{-2} \cdot f_{1}(Z(t-u))\right] \\
& \widetilde{S}_{Z}^{(\alpha)}=2 \mathcal{J}\left[(\max (\alpha, t)-\min (\alpha, u))^{-2} \cdot f_{1}(Z(\max (\alpha, t)-\min (\alpha, u))] .\right.
\end{aligned}
$$

Proof. The probability that $M\left(u^{\prime}\right)$ and $M\left(t^{\prime}\right)$ are both keys for some $u^{\prime} \in[u, u+d u]$ and $t^{\prime} \in[t, t+d t]$ is $Z^{2} d u d t$. Denote by $[x, y]:=[x(u, t), y(u, t)]$ the smallest closed interval that contains $\{u, t, \alpha\}$ (QuickVal $\alpha$ ) or $\{u, t\}$ (QuickSort). Conditionally, given 
that $M(u)$ and $M(t)$ are both keys, $M(u)$ and $M(t)$ are compared if and only if $M(u)$ or $M(t)$ is chosen as the pivot amongst the set $\mathcal{M}:=\{M(z) ; z \in[x, y]\}$. The cardinality of the "good" set $\{M(u), M(t)\}$ is 2 , while the cardinality of $\mathcal{M}$ equals $2+N[x, y]$, where $N[x, y]$ is the number of keys strictly between $M(x)$ and $M(y)$. Then, for any fixed set of words, the probability that $M(u)$ and $M(t)$ are compared is $2 /(2+N[x(u, t), y(u, t)])$. To evaluate the mean value of this ratio in the Poisson model, we remark that, if we draw Poi $(Z)$ i.i.d. random variables uniformly distributed over $[0,1]$, the number $N(\lambda)$ of those that fall in an interval of (Lebesgue) measure $\lambda$ is Poi $(\lambda Z)$ distributed, so that

$$
\mathbb{E}\left[\frac{2}{N(\lambda)+2}\right]=\sum_{k \geq 0} \frac{2}{k+2} e^{-\lambda Z} \frac{(\lambda Z)^{k}}{k !}=\frac{2}{\lambda^{2} Z^{2}} f_{1}(\lambda Z) .
$$

1.5. Exact formulae for $S_{n}$. We now return to the model of prime interest, where the number of keys is a fixed number $n$.

Proposition 3. Assume that $\Lambda(s)$ of $(7)$ converges at $s=-2$. Then the mean values $S_{n}$, associated with QuickSort and QuickVal ${ }_{\alpha}$ can be expressed as

$$
S_{n}=\sum_{k=2}^{n}\left(\begin{array}{l}
n \\
k
\end{array}\right)(-1)^{k} \varpi(-k), \quad \text { for } n \geq 2,
$$

where $\varpi(s)$ is a series of Dirichlet type given, respectively, by

$$
\varpi(s)=2 \mathcal{J}\left[(t-u)^{-(2+s)}\right], \quad \varpi(s)=2 \mathcal{J}\left[(\max (\alpha, t)-\min (\alpha, u))^{-(2+s)}\right] .
$$

The two functions $\varpi(s)$ are defined for $\Re s \leq-2$; they depend on the algorithm and the source and are called the mixed Dirichlet series.

For QuickSort, the series $\varpi(s)$ admits a simple form in terms of $\Lambda(s)$ :

$$
\varpi(s)=\frac{\Lambda(s)}{s(s+1)}, \quad S_{n}=2 \sum_{k=2}^{n} \frac{(-1)^{k}}{k(k-1)}\left(\begin{array}{l}
n \\
k
\end{array}\right) \sum_{w \in \Sigma^{*}} p_{w}^{k} .
$$

For QuickVal, similar but more complicated forms can be obtained.

Proof (Prop. 3). Consider any sequence $S_{n}$ whose Poissonized version is of the form

$$
\widetilde{S}_{Z} \equiv e^{-Z} \sum_{n \geq 0} S_{n} \frac{Z^{n}}{n !}=\int_{D} \lambda(x) f_{1}(Z \mu(x)) d x
$$

for some domain $D \subset \mathbb{R}^{d}$ and some weights $\lambda(x), \mu(x) \geq 0$, with, additionally, $\mu(x) \leq 1$. By expanding $f_{1}$, then exchanging the order of summation and integration, one obtains

$$
\widetilde{S}_{Z}=\sum_{k=2}^{\infty}(-1)^{k} \varpi(-k) \frac{Z^{k}}{k !}, \quad \text { where } \varpi(-k):=\int_{D} \lambda(x) \mu(x)^{k} d x .
$$

Analytically, the form (10) is justified as soon as the integral defining $\varpi(-2)$ is convergent. Then, since $S_{n}$ is related to $\widetilde{S}_{Z}$ via the relation $S_{n}=n !\left[Z^{n}\right]\left(e^{Z} \widetilde{S}_{Z}\right)$, it can be recovered by a binomial convolution.

\section{$2 \quad$ Asymptotic analysis}

For asymptotic purposes, the singular structure of the involved functions, most notably the mixed Dirichlet series $\varpi(s)$, is essential. With tameness assump- 
tions described in Subsection 2.1, it becomes possible to develop complex integral representations (Subsection 2.2), themselves strongly tied to Mellin transforms $[9,18]$. We can finally conclude with the proofs of Theorems 1 and 2 in Subsection 2.3.

2.1. Tamed sources. We now introduce important regularity properties of a source, via its Dirichlet series $\Lambda(s), \Pi(s)$, as defined in (7). Recall that $\Lambda(s)$ always has a singularity at $s=-1$, and $\Pi(s)$ always has a singularity at $s=0$.

Definition 3. Consider a source $\mathcal{S}$, with $\Lambda(s), \Pi(s)$ its Dirichlet series.

(a) The source $\mathcal{S}$ is $\Pi$-tamed if the sequence $\pi_{k}$ satisfies $\pi_{k} \leq A k^{-\gamma}$ for some $A>0, \gamma>1$. In this case, $\Pi(s)$ is analytic for $\Re(s)<-1 / \gamma$.

(b) The source $\mathcal{S}$ is $\Lambda$-tamed if $\Lambda(s)$ is meromorphic in a "hyperbolic domain"

$$
\mathcal{F}=\left\{s \quad \mid \Re s \leq-1+\frac{c}{(1+|\Im s|)^{d}}\right\} \quad(c, d>0),
$$

is of polynomial growth as $|s| \rightarrow \infty$ in $\mathcal{F}$, and has only a pole at $s=-1$, of order 1 , with a residue equal to the inverse of the entropy $h(\mathcal{S})$.

(c) The source $\mathcal{S}$ is $\Lambda$-strongly tamed if $\Lambda(s)$ is meromorphic in a half-plane

$$
\mathcal{F}=\left\{\Re(s) \leq \sigma_{1}\right\}
$$

for some $\sigma_{1}>-1$, is of polynomial growth as $|s| \rightarrow \infty$ in $\mathcal{F}$, and has only a pole at $s=-1$, of order 1 , with a residue equal to the inverse of the entropy $h(\mathcal{S})$.

$(d)$ The source $\mathcal{S}$ is periodic if $\Lambda(s)$ is analytic in $\Re(s)<-1$, has a pole of order 1 at $s=-1$, with a residue equal to the inverse of the entropy $h(\mathcal{S})$, and admits an imaginary period $i \Omega$, that is, $\omega(s)=\omega(s+i \Omega)$.

Essentially all "reasonable" sources are $\Pi$-tamed and "most" classical sources are $\Lambda$-tamed; see Section 3 for a discussion. The properties of $\varpi(s)$ turn out to be related to properties of the source via the Dirichlet series $\Lambda(s), \Pi(s)$.

Proposition 4. The mixed series $\varpi(s)$ of QuickVal $\alpha$ relative to a $\Pi$-tamed source with exponent $\gamma$ is analytic and bounded in $\Re(s) \leq-1+\delta$ with $\delta<1-1 / \gamma$. The mixed series $\varpi(s)$ of QuickSort is meromorphic and of polynomial growth in a hyperbolic domain (11) when the source is $\Lambda$-tamed, and in a vertical strip (12) when the source is $\Lambda$-strongly tamed.

2.2. General asymptotic estimates. The sequence of numerical values $\varpi(-k)$ lifts into an analytic function $\varpi(s)$, whose singularities essentially determine the asymptotic behaviour of QuickSort and QuickSelect.

Proposition 5. Let $\left(S_{n}\right)$ be a numerical sequence which can be written as

$$
S_{n}=\sum_{k=2}^{n}\left(\begin{array}{l}
n \\
k
\end{array}\right)(-1)^{k} \varpi(-k), \quad \text { for } n \geq 2 .
$$

(i) If the function $\varpi(s)$ is analytic in $\Re(s)<C$, with $-2<C<-1$, and is of polynomial growth with order at most $r$, then the sequence $S_{n}$ admits a 
Nörlund-Rice representation, for $n>r+1$ and any $-2<d<C$ :

$$
S_{n}=\frac{1}{2 i \pi} \int_{d-i \infty}^{d+i \infty} \varpi(s) \frac{n !}{s(s+1) \cdots(s+n)} d s .
$$

(ii) If, in addition, $\varpi(s)$ is meromorphic in $\Re(s)<D$ for some $D>-1$, has only a pole (of order $k_{0} \geq 0$ ) at $s=-1$ and is of polynomial growth in $\Re(s)<D$ as $|s| \rightarrow+\infty$, then $S_{n}$ satisfies $S_{n}=A_{n}+O\left(n^{1-\delta}\right)$, for some $\delta>0$, with

$$
A_{n}=-\operatorname{Res}\left(\frac{n ! \varpi(s)}{s(s+1) \cdots(s+n)} ; s=-1\right)=n\left(\sum_{k=0}^{k_{0}} a_{k} \log ^{k} n\right) \text {. }
$$

2.3. Application to the analysis of the three algorithms. We conclude our discussion of algorithms QuickSort and QuickVal, then that of QuickQuant.

- Analysis of QuickSort. In this case, the Dirichlet series of interest is

$$
\frac{\varpi(s)}{s+1}=\frac{2}{s+1} \mathcal{J}\left[(t-u)^{-(2+s)}\right]=2 \frac{\Lambda(s)}{s(s+1)^{2}} .
$$

For a $\Lambda$-tamed source, there is a triple pole at $s=-1$ : a pole of order 1 arising from $\Lambda(s)$ and a pole of order 2 brought by the factor $1 /(s+1)^{2}$. For a periodic source, the other poles located on $\Re s=-1$ provide the periodic term $n P(\log n)$, in the form of a Fourier series. This concludes the proof of Theorem 1.

- Analysis of QuickVal ${ }_{\alpha}$. In this case, the Dirichlet series of interest is

$$
\frac{\varpi(s)}{s+1}=\frac{2}{s+1} \mathcal{J}\left[(\max (\alpha, t)-\min (\alpha, u))^{-(2+s)}\right] .
$$

Proposition 4 entails that $\varpi(s)$ is analytic at $s=-1$. Then, the integrand in (13) has a simple pole at $s=-1$, brought by the factor $1 /(s+1)$ and Proposition 5 applies as soon as the source $\mathcal{S}$ is $\Pi$-tamed. Thus, for $\delta<1-(1 / \gamma)$ :

$$
V_{n}^{(\alpha)}=\rho_{\mathcal{S}}(\alpha) n+O\left(n^{1-\delta}\right)
$$

The three possible expressions of the function $(u, t) \mapsto \max (\alpha, t)-\min (\alpha, u)$ on the unit triangle give rise to three intervals of definition for the function $H$ defined in Figure 1 (respectively, ]- $\infty,-1 / 2]$, [-1/2,+1/2], [1/2, $\infty[$ ).

- Analysis of QuickQuant ${ }_{\alpha}$. The main chain of arguments connecting the asymptotic behaviors of QuickVal ${ }_{\alpha}(n)$ and QuickQuant ${ }_{\alpha}(n)$ is the following.

(a) The algorithms are asymptotically "similar enough": If $X_{1}, \ldots, X_{n}$ are $n$ i.i.d. random variables uniform over $[0,1]$, then the $\alpha$-quantile of set $X$ is with high probability close to $\alpha$. For instance, it is at distance at $\operatorname{most}^{2}\left(\log ^{2} n\right) / \sqrt{n}$ from $\alpha$ with an exponentially small probability (about $\exp \left(-\log ^{2} n\right)$ ).

(b) The function $\alpha \mapsto \rho_{\mathcal{S}}(\alpha)$ is Hölder with exponent $c=\min (1 / 2,1-(1 / \gamma))$.

(c) The error term in the expansion (15) is uniform with respect to $\alpha$.

\section{Sources, QuickSort, and QuickSelect}

We can now recapitulate and examine the situation of classical sources with respect to the tameness and periodicity conditions of Definition 3. All the sources listed below are $\Pi$-tamed, so that Theorem 2 (for QuickQuant) is systematically 
applicable to them. The finer properties of being $\Lambda$-tamed, $\Lambda$-strongly tamed, or periodic only intervene in Theorem 1, for Quicksort.

Memoryless sources and Markov chains. The memoryless (or Bernoulli) sources correspond to an alphabet of some cardinality $r \geq 2$, where symbols are taken independently, with $p_{j}$ the probability of the $j$ th symbol. The Dirichlet series is

$$
\Lambda(s)=\left(1-p_{1}^{-s}-\cdots-p_{r}^{-s}\right)^{-1}
$$

Despite their simplicity, memoryless sources are never $\Lambda$-strongly tamed. They can be classified into three subcategories (Markov chain sources obey a similar trichotomy).

(i) A memoryless source is said to be Diophantine if at least one ratio of the form $\left(\log p_{i}\right) /\left(\log p_{j}\right)$ is irrational and poorly approximable by rationals. All Diophantine sources are $\Lambda$-tamed in the sense of Definition 3(b); i.e., the existence of a hyperbolic region (11) can be established. Note that, in a measure-theoretic sense, almost all memoryless sources are Diophantine.

(ii) A memoryless source is periodic when all ratios $\left(\log p_{i}\right) /\left(\log p_{1}\right)$ are rational (this includes cases where $p_{1}=\cdots=p_{r}$, in particular, the model of uniform random bits). The function $\Lambda(s)$ then admits an imaginary period, and its poles are regularly spaced on $\Re(s)=-1$. This entails the presence of an additional periodic term in the asymptotic expansion of the cost of QuickSort, which corresponds to complex poles of $\Lambda(s)$; this is Case (ii) of Theorem 1.

(iii) Finally, Liouvillean sources are determined by the fact that the ratios are not all rational, but all the irrational ratios are very well approximated by rationals (i.e., have infinite irrationality measure); for their treatment, we can appeal to the Tauberian theorems of Delange [6]

Dynamical sources were described in Section 1. They are principally determined by a shift transformation $T$. As shown by Vallée [19] (see also [3]), the Dirichlet series of fundamental intervals can be analysed by means of transfer operators, specifically the ones of secant type. We discuss here the much researched case where $T$ is a non-linear expanding map of the interval. (Such a source may be periodic only if the shift $T$ is conjugated to a piecewise affine map of the type previously discussed.) One can then adapt deep results of Dolgopyat [4], to the secant operators. In this way, it appears that a dynamical source is strongly tamed as soon as the branches of the shift (i.e., the restrictions of $T$ to the intervals $\mathcal{I}_{\sigma}$ ) are not "too often" of the "same form" - we say that such sources are of type $D 1$. Such is the case for continued fraction sources, which satisfy a "Riemann hypothesis" [1,2]. The adaptation of other results of Dolgopyat [5] provides natural instances of tamed sources with a hyperbolic strip: this occurs as soon as the branches all have the same geometric form, but not the same arithmetic features - we say that such sources are of type $D 2$.

Theorem 3. The memoryless and Markov chain sources that are Diophantine are 1-tamed. Dynamical sources of type D2 are 1 -tamed and dynamical sources of type D1 are 4 -strongly tamed. The estimates of Theorem 1 and 2 are then applicable to all these sources, and, in the case of $\Lambda$-strongly tamed sources, the error term of QuickSort in (1) can be improved to $O\left(n^{1-\delta}\right)$, for some $\delta>0$. 
It is also possible to study "intermittent sources" in the style of what is done for the subtractive Euclidean algorithm [20, $\$ 2,6]$ : a higher order pole of $\Lambda(s)$ then arises, leading to complexities of the form $n \log ^{3} n$ for QuickSort, whereas QuickQuant remains of order $n$.

Acknowledgements. The authors are thankful to Také Nakama for helpful discussions. Research for Clément, Flajolet, and Vallée was supported in part by the SADA Project of the ANR (French National Research Agency). Research for Fill was supported by The Johns Hopkins University's Acheson J. Duncan Fund for the Advancement of Research in Statistics. Thanks finally to Jérémie Lumbroso for a careful reading of the manuscript.

\section{References}

1. Baladi, V., AND VAllÉE, B. Euclidean algorithms are Gaussian. Journal of Number Theory 110 (2005), 331-386.

2. BAladi, V., AND VAllÉE, B. Exponential decay of correlations for surface semiflows without finite Markov partitions. Proc. AMS 133, 3 (2005), 865-874.

3. Clément, J., Flajolet, P., And Vallée, B. Dynamical sources in information theory: A general analysis of trie structures. Algorithmica 29, 1/2 (2001), 307-369.

4. Dolgopyat, D. On decay of correlations in Anosov flows, Annals of Mathematics 147 (1998) 357-390.

5. Dolgopyat, D. Prevalence of rapid mixing (I) Ergodic Theory and Dynamical Systems 18 (1998) 1097-1114.

6. Delange, H. Généralisation du théorème de Ikehara. Annales scientifiques de l'École Normale Supérieure Sér. 3 71, 3 (1954), 213-142.

7. Fill, J. A., AND JANSON, S. The number of bit comparisons used by Quicksort: An average-case analysis. In Proceedings of the ACM-SIAM Symposium on Discrete Algorithms (SODA04) (2001), pp. 293-300.

8. FILL, J. A., AND NAKAMA, T. Analysis of the expected number of bit comparisons required by Quickselect. ArXiv:0706.2437. To appear in Algorithmica. Extended abstract in Proceedings ANALCO (2008), SIAM Press, 249-256.

9. Flajolet, P., And Sedgewick, R. Mellin transforms and asymptotics: finite differences and Rice's integrals. Theoretical Comp. Sc. 144 (1995), 101-124.

10. Flajolet, P., And Sedgewick, R. Analytic Combinatorics. Cambridge University Press, 2009. Available electronically from the authors' home pages.

11. Grabner, P., And Prodinger, H. On a constant arising in the analysis of bit comparisons in Quickselect. Quaestiones Mathematicae 31 (2008), 303-306.

12. Kirschenhofer, P., Prodinger, H., And Martínez, C. Analysis of Hoare's FIND Algorithm. Random Structures \&5 Algorithms. 10 (1997), 143-156.

13. KNuth, D. E. The Art of Computer Programming, 2nd ed., vol. 3: Sorting and Searching. Addison-Wesley, 1998.

14. NörLund, N. E. Leçons sur les équations linéaires aux différences finies. GauthierVillars, Paris, 1929

15. NÖRLund, N. E. Vorlesungen über Differenzenrechnung. Chelsea Publishing Company, New York, 1954.

16. Sedgewick, R. Quicksort. Garland Pub. Co., New York, 1980. Reprint of Ph.D. thesis, Stanford University, 1975.

17. Sedgewick, R. Algorithms in C, Parts 1-4, third ed. Addison-Wesley, Reading, Mass., 1998.

18. SzPankowski, W. Average-Case Analysis of Algorithms on Sequences. John Wiley, 2001.

19. VAllée, B. Dynamical sources in information theory: Fundamental intervals and word prefixes. Algorithmica 29, 1/2 (2001), 262-306.

20. VAllée, B. Euclidean dynamics. Discrete and Continuous Dynamical Systems 15, 1 (2006), 281-352. 